\title{
Die Teeplantagen Sri Lankas - Gestalt und Wandel eines monokulturellen Wirtschaftsraumes
}

Die Teewirtschaft Sri Lankas, wie Ceylon seit 1972 offiziell heißt, bestimmt seit über einhundert Jahren das wirtschaftliche und weithin auch das wirtschaftsräumliche Gefüge der $65000 \mathrm{~km}^{2}$ messenden Insel. Selten hat die Plantage, ein «landwirtschaftlicher Großbetrieb, der vorwiegend pflanzliche Produkte für den Markt liefert», einen Wirtschaftsraum so intensiv geprägt wie im Zentralen Bergland von Sri Lanka. Da sich die Plantagen in unbesiedeltem, von montanem Regenwald überwuchertem Bergland ausbreiteten, konnte eine Transformation der Naturlandschaft in eine Kulturlandschaft erfolgen, in der alle Elemente nach streng funktionalen Kriterien im Hinblick auf die monokulturelle Nutzung des Raums geordnet waren, lediglich durch naturräumliche Gegebenheiten wie Klima, Boden und Relief beschränkt. Aufgrund der starken Zergliederung des Gebirgsmassivs können jedoch nur wenige Anbaugebiete als geschlossene monokulturelle Plantagenwirtschaftsräume größeren Ausmaßes angesprochen werden, so etwa das Hatton Plateau, das Gebiet um Haputale oder das Tal von Uda Pussalawa bei Nuwara Eliya.

Nicht nur das Vorherrschen eines Anbauproduktes, sondern das gesamte wirtschaftsräumliche Gefüge verleihen diesen Räumen besondere unverwechselbare Züge.

Nahezu lückenlos überziehen die Teefelder Berge und Täler, durchwoben vom engmaschigen Netz der Wirtschaftswege. Selbst Reste ursprünglicher Vegetation dicht unterhalb der Gipfel sind als Brennstoffquellen in den Produktionsprozeß mit einbezogen. Zentrales Gebäude der Teelandschaft ist die mehrstöckige Fabrik, in der die Umwandlung vom grünen Blatt zum konsumfertigen Produkt stattfindet. Die zahlreichen Stockwerke deuten noch auf den bis vor kurzem üblichen raumbeanspruchenden Trocknungsprozeß (Welken), bei dem die grünen Blätter in dünnen Lagen auf Netzen ausgebreitet wurden. Vielerorts ist er bereits durch ein neues zeit- und raumsparendes Verfahren (Trog-Welken) ersetzt, so $\mathrm{da} ß$ die oberen Stockwerke nun meist leerstehen. Den überwiegenden Anteil der Bevölkerung stellen die Tamilen (in Nuwara Eliya über $80 \%$ ), die während der letzten einhundert Jahre in Südindien als Plantagenarbeiter angeworben worden sind und zum Teil bereits seit mehreren Generationen im Lande leben, ohne allerdings die Staatsbürgerschaft erhalten zu haben. Die Arbeiter wohnen in den kuli-lines, langgestreckten flachen Gebäuden (s. Bildmitte unten), in denen jeder Familie ein Raum zugewiesen ist. Unterkunft, Versorgung mit Grundnahrungsmitteln und Sozialleistungen obliegen der Plantage.

Äußerer Ausdruck strenger Hierarchie sind die luxuriösen Managerbungalows, die in bevorzugter Lage die Landschaft überblicken. Die englischen Plantagenleiter haben jedoch zum großen Teil das Land verlassen, wenn auch noch etwa $30 \%$ der Unternehmen in ausländischem Besitz sind. Als weitere Siedlungsform beherrscht der Bazar das Bild des Teehochlandes. Meist verkehrsgünstig an einer Straße gelegen, nimmt er die Versorgung der umliegenden Plantagen mit Dienstleistungen und einem speziell auf die Plantagenarbeiter abgestimmten Warensortiment wahr. Kennzeichnend sind die Goldschmiede, die die Vorliebe der Tamilen für die Kapitalanlage in Edelmetallen bezeugen, sowie die Büros der sehr aktiven Gewerkschaften. Viele der Geschäfte sind in Hand von Mohammedanern (moors). Bedingt durch die geringe Kaufkraft der Plantagenarbeiter und fehlende Ausbreitungsmöglichkeiten sind die Bazarorte nur klein und liegen weitgestreut (nur wenige zählen über 2000 Einwohner).

Seit Beginn der Unabhängigkeit ist eine zunehmende Diversifikation der Landnutzung in den monokulturellen Agrarräumen zu verzeichnen. Sie dokumentiert die Bemühungen des Landes, die mit der Monokultur verbundene Weltmarktabhängigkeit zu beseitigen.

Begünstigt durch klimatische und wirtschaftliche Faktoren hat sich besonders um Nuwara Eliya ein intensiver marktorientierter Gemüseanbau entwikkelt. Auch die Milchwirtschaft gewinnt zunehmend an Bedeutung. Fast $2 / 3$ der Milchproduktion der Insel entstammen dem Teehochland, wo das Vieh in Ställen auf den Plantagen gehalten wird.

Verglichen mit den übrigen Wirtschaftsräumen, wo sich traditionelle Agrarwirtschaft und weltmarktorientierter Plantagenbau verzahnen, sind die monokulturellen Anbaugebiete dennoch durch eine bemerkenswerte Stabilität gekennzeichnet, die nicht zuletzt auf die nach wie vor zentrale Bedeutung der Teepflanze für die Wirtschaft Sri Lankas zurückzuführen ist.

H. J. Aubert, Augustastr. 83, D-5300 Bonn - Bad Godesberg 


\section{Literatur}

COOK, E. K.: Ceylon, its Geography, its Resources and its People, London 1951, 2. Aufl.

COORAY, P. G.: An Introduction to the Geology of Ceylon, Colombo 1967

FORREST, D. M.: A hundred Years of Ceylon Tea, London 1967

MANSHARD, w.: Einführung in die Agrargeographie der Tropen, Mannheim 1968
SCHWEINFURTH, U.: Die Teelandschaft im Hochland der Insel Ceylon als Beispiel für den Landschaftswandel, Wiesbaden 1966

SCHWEINFURTH, U.: Landschaftsökologische Forschungen auf der Insel Ceylon, Wiesbaden 1971

SIEVERS, A.: Ceylon. Gesellschaft und Wirtschaftsraum in den Tropen, Wiesbaden 1964

TIMMERMANN, O. F.: Ceylon, seine natürlichen Landschaftsbilder und -typen, München 1935

\section{Literaturbesprechung}

LOEFFLER E.: Untersuchungen zum eiszeitlichen und rezenten klimagenetischen Formenschatz in den Gebirgen Nordostanatoliens, Heidelberger Geographische Arbeiten, 27, 1970.

Dieser als Dissertation ausgeführten Untersuchung liegt ein für die vergleichende Hochgebirgsmorphologie besonders interessantes Arbeitsgebiet zugrunde: Es liegt "zwischen der feuchten Schwarzmeerküste und dem trockenen anatolischen Hochland, zwischen dem Kaukasus und den mediterranen Gebirgen" (p. 11). - Der Autor befasst sich zuerst mit dem Problem der eiszeitlichen und nacheiszeitlichen Vergletscherung und wendet sich anschliessend der periglazialen Höhenstufe zu (Formen und Vorgänge solifluidaler und nicht solifluidaler Natur, Hangsymmetrie).

Verschiedene Autoren versuchten, die hohe Lage der eiszeitlichen Schneegrenze der küstennahen Pontischen Gebirgsketten mit einer postglazialen Hebung des gesamten Gebirgskörpers zu erklären. Um diese Hypothese überprüfen zu können, wandte sich Löffler dem Studium des Verlaufes junger Schwarzmeerküstenterrassen $\mathrm{zu}$ und legt seine Ergebnisse in einem dritten Kapitel vor. Er kam zum Schluss, dass "keine stark verstellten oder gehobenen jungen Küstenterrassen" beobachtet werden können (p. 110). Auch eine Gegenüberstellung der eiszeitlichen und rezenten Schneegrenzenhöhe spricht gegen postglaziale Hebungsvorgänge. Die hohe eiszeitliche Schneegrenze im Ostanatolischen Gebirge erklärt der Verfasser daher mit "der Tatsache, dass das Hochgebirge nicht unter dem Einfluss des Küstenklimas steht, sondern viel stärker vom Wettergeschehen des Binnenlandes beherrscht wird" (p. 108).

Die vorliegende Publikation wird ihrer Zielsetzung - Feststellen des eiszeitlichen und rezenten klimatisch-geomorphologischen Formenschatzes (p. 11) - in hohem Masse gerecht. Es liegt wohl in der Lage des Untersuchungsgebietes begründet, dass die Arbeit ohne Einsatz moderner Feld- und Labormethoden durchgeführt werden musste.

G. Furrer 